\title{
Developing cardiac rupture as initial sign of acute myocardial infarction
}

\author{
J-P. van Mantgem ${ }^{1}$ and A. E. Becker \\ From the Department of Pathology, University of Amsterdam, Wilhelmina Gasthuis, Amsterdam, The Nether- \\ lands
}

Pathological data are given of 4 patients in whom the development of cardiac rupture was the initial sign of myocardial infarction. The term 'concealed cardiac rupture' is proposed for this condition. In each instance the clinical diagnosis was 'onset of infarction', whereas the necropsy suggested that the acute onset of symptoms was caused by the start of rupture.

The arguments for classifying the cases as 'concealed rupture' are (1) the lack of correlation between histological and clinical dating of the infarct, (2) the observation that the tear may take considerable time before actual epicardial breakthrough occurs, and (3) the fact that a high percentage of infarcts may pass clinically unnoticed.

Cardiac tamponade is at present an important cause of death in patients with acute myocardial infarction. At the coronary care unit of the department of cardiology an incidence of 27 per cent was found among patients who died of acute infarction (Lie, Roels-van Ijsseldijk, and Van Capelle, 1975). To the clinician the occurrence of cardiac rupture is often sudden and unexpected, since definite warning signs are not present. However, morphologists have previously suggested that the heart specimen often shows features indicative of a delayed rupture (Lautsch and Lanks, 1967). It has also been stated that occasionally a patient may present with symptoms of acute infarction, which actually represented the start of the rupture (Lautsch and Lanks, 1967; Van Tassel and Edwards, 1972). In a recent correlative study of 50 patients with cardiac tamponade this was confirmed (Becker and van Mantgem, 1975). The term 'concealed rupture' was proposed to indicate the circumstance in which such a lack of correlation existed between clinical symptomatology and morphological features. It is the purpose of this report to re-emphasize the occurrence of this phenomenon.

\section{Received 8 December 1975.}

1Army reserve Medical Officer stationed at the Department of Pathology, University of Amsterdam, Wilhelmina Gasthuis.
Case 1

A 61-year-old woman was known to have hypertension for 7 years. She first complained of pain in the left arm after a minor traffic accident. Two days later she developed vague pain in the chest. However, after two more days she suddenly experienced severe chest pain and she collapsed. On immediate admission she was sweating. Physical examination revealed a systolic blood pressure of $70 \mathrm{mmHg}$ and a heart rate of 110 per minute. The central venous pressure judged from the jugular veins was not raised. The electrocardiogram showed the signs of recent anterolateral myocardial infarction. She rapidly developed signs of cardiogenic shock. She was treated with lignocaine, but no anticoagulants were given. Sixteen hours after admission she suddenly died.

The necropsy showed a large transmural myocardial infarction in the anteroseptal wall of the left ventricle. It showed a central rupture which had led to a tamponade of $400 \mathrm{ml}$ blood. At the site of rupture there was a distinct erosion of the necrotic myocardium (Fig. 1A). The actual tear was slit-like and located at the top of the erosion. Thrombus was covering the site of rupture and part of the eroded area. There was two-vessel disease of the coronary arteries, with total recent occlusion of both the anterior descending artery 


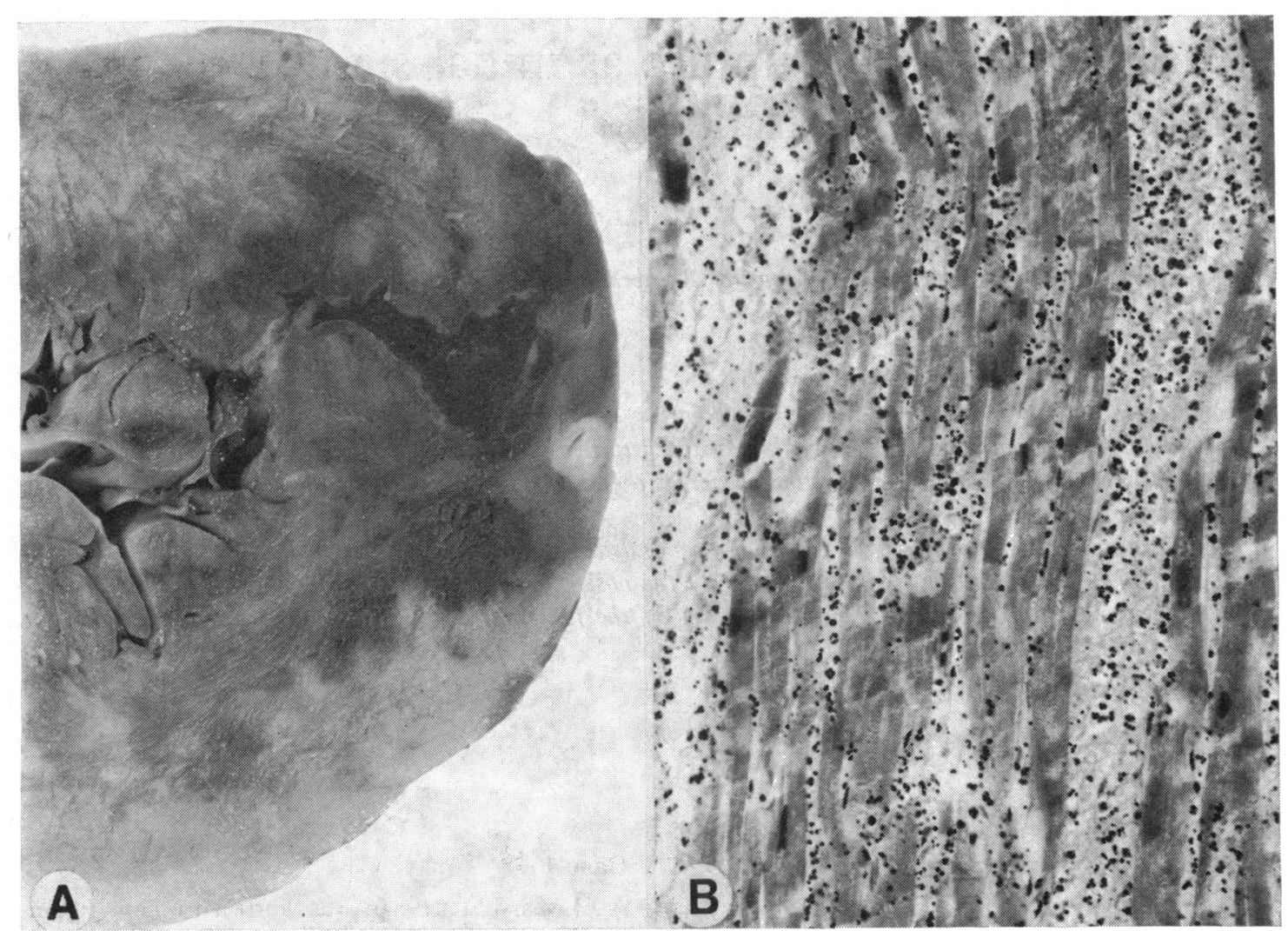

FIG. 1 Case 1. The clinical dating of the infarct was 16 hours. (A) The gross aspect of part of the anterolateral wall of the left ventricle. The transmural infarct is seen. At the site of rupture an erosion of the affected myocardium has occurred. The actual epicardial breakthrough cannot be seen. (B) A representative microscopical section of the infarcted myocardium. There is massive infiltration of dead myocardium by polymorphonuclear leucocytes. This is not in agreement with an infarct of 16 hours' duration but, instead, suggests that the vague complaints two days before admission represented the onset of infarction. The erosion at the site of rupture indicates a slowly progressive tear. Therefore, the sudden onset of severe chest pain could have been caused by the onset of the rupture process. (Haematoxylin and eosin. $\times 200$.)

and the left circumflex branch.

Microscopical sections of the infarct showed a massive leucocytic infiltration of the necrotic myocardium, indicative of a myocardial infarction of a much older date than the 24 hours indicated by the clinical history (Fig. 1B). In retrospect, therefore, this patient probably experienced an infarction after her minor traffic accident, while the acute episode in effect represented the onset of rupture.

\section{Case 2}

A 53-year-old man had complained for some weeks of intermittent chest pain, occasionally radiating towards the left arm. The pain was always related to periods of emotional disturbance. Four days before death he woke up in the middle of the night with severe chest pain, radiating to the left arm and accompanied by heavy sweating. He was admitted to hospital 4 hours later and on examination showed a blood pressure of $160 / 110 \mathrm{mmHg}$, with a heart rate of 100 per minute. His central venous pressure was not raised, as judged from the neck veins. The electrocardiogram showed recent inferior wall infarction. He was treated with lignocaine and he was given anticoagulants. The pain disappeared. The next day he developed signs of acute abdominal distress, for which no conclusive explanation was found. Three days later, that is 4 

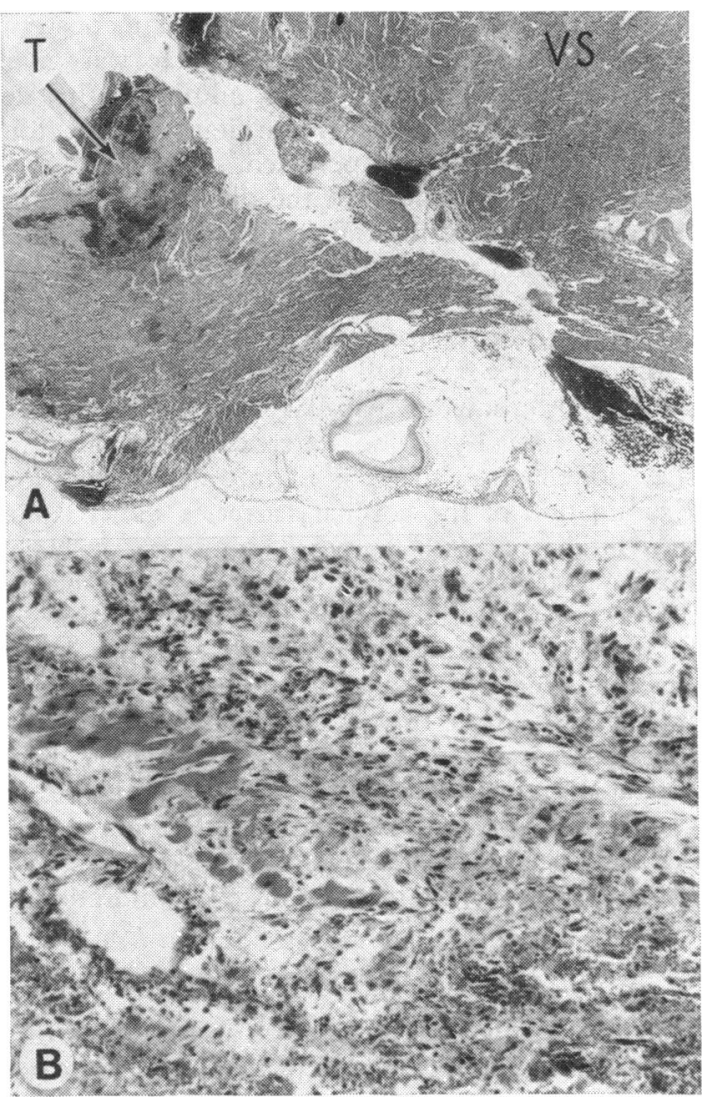

FIG. 2 Case 2. The clinical dating of the infarct was 4 days. (A) Microscopical section of the tear in the posterior wall of the left ventricle, at the junction with the ventricular septum (VS). The site of rupture shows an erosion at the inner aspect of the affected myocardium covered by thrombus $(T)$. (B) $A$ detailed view of a representative section of the infarct. It is characterized by a proliferation of fibroblasts and capillaries, indicative of an early reparative response. A recent haemorrhage, secondary to the dissecting tear, is present. These features are not in agreement with an infarct of 4 days but, instead, fit an infarct of between 1 and 3 weeks old. In retrospect this could correlate with the patient's history. (Haematoxylin and cosin. $A \times 4 \cdot 7, B \times 165$.)

days after admission, he suddenly died.

The necropsy confirmed the presence of a large transmural inferior wall infarct, which extended from the apex to the base of the heart and also involved the posteromedial papillary muscle group. The heart had ruptured posteriorly, close to the ventricular septum (Fig. 2A). There was a tamponade of $500 \mathrm{ml}$ blood. The coronary arteries

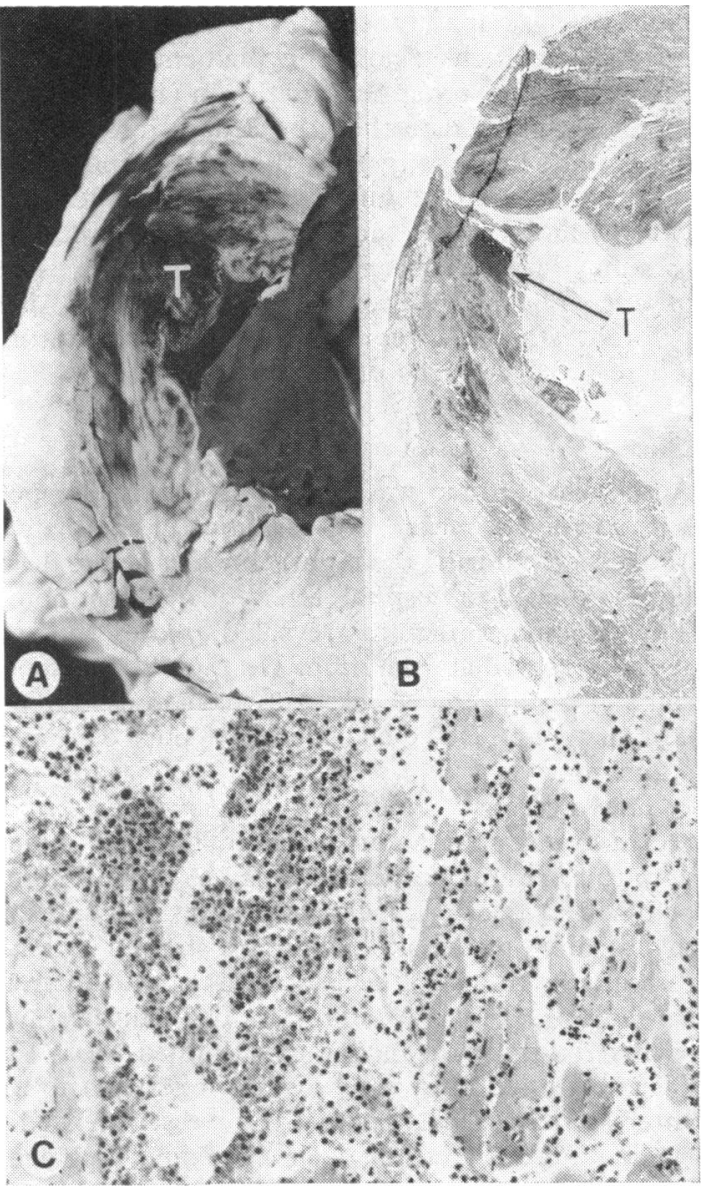

FIG. 3 Case 3. The clinical dating of the infarct was 24 hours. (A) The gross aspect of the rupture site in a transmural anteroseptal myocardial infarct. The area shows a distinct erosion, covered with thrombus $(T)$. The rupture is located centrally in the infarct. (B) $A$ microscopical view of the same area in which the localized loss of myocardium and subsequent covering by thrombus $(T)$ is again seen. (C) A representative microscopical section of the infarct. Thsre is massive infiltration by polymorphonuclear leucocytes, indicative of an infarct older than 24 hours. (Haematoxylin and eosin. $B \times 2 \cdot 0, C \times 130$.)

showed two-vessel disease, with total occlusions distally in the right coronary artery and proximally in the anterior descending branch. There were no signs of previous myocardial infarctions.

The microscopy of the myocardium showed signs of an early reparative response with ingrowth of fibroblasts and capillaries (Fig. 2B). The cellular 
infiltrate was mainly composed of macrophages, carrying lipofuchsin and iron pigments, and these changes were consistent with an infarct of a somewhat older date than the 4 days judged from the clinical history. In fact, the histological dating suggests myocardial infarction of somewhere between 1 and 3 weeks earlier. Again the distinct discrepancy suggested concealed rupture, in a silent infarct. The sudden onset of symptoms 4 days before death could represent the onset of rupture. There were no signs of acute infarction.

\section{Case 3}

A 70-year-old man was known to have had hypertension for two years. He occasionally complained of chest pain during the night. One day before death he was rushed to hospital, after collapsing at home. The electrocardiogram revealed recent anteroseptal myocardial infarction. He rapidly developed signs of cardiogenic shock. Anticoagulants were not administered. He died suddenly one day after admission.

The necropsy confirmed the presence of a recent transmural anteroseptal myocardial infarct with rupture and tamponade (Fig. 3A). The amount of blood in the pericardial cavity was $300 \mathrm{ml}$. The myocardium at the site of rupture showed extreme thinning, with loss of necrotic muscle and adherent thrombosis (Fig. 3A and B). The rupture had occurred through a moderately thinned area. The heart exhibited one-vessel disease, restricted to the anterior descending artery. The vessel showed a recent total occlusion in its proximal segment. The aortic valve was slightly calcified, complicating a congenitally bicuspid valve. The left ventricle was very hypertrophic.

Microscopical studies of the myocardium showed a massive infiltration with polymorphonuclear leucocytes, indicative of myocardial infarction of more than one day's duration (Fig. 3C). Based upon the microscopical studies the 'age' of the infarct was estimated as between 3 and 7 days. The morphological aspects, therefore, suggest that this was another example of concealed rupture. The sudden clinical onset probably represented the onset of rupture in the setting of an unrecognized infarction.

\section{Case 4}

A 54-year-old man suddenly developed chest pain accompanied by nausea and sweating 5 days before death. The pain disappeared spontaneously one day later. Three days later, that is one day before death, he suddenly collapsed while shaving. On immediate admission he had signs of cardiogenic shock. The electrocardiogram showed acute posteroinfero- and lateral wall infarction. He was given anticoagulants. One day after admission he developed signs of mitral regurgitation, and four hours later he suddenly perished.

The necropsy confirmed the diagnosis of a large transmural posteroinferior wall infarct extending into the lateral wall of the left ventricle (Fig. 4A). A rupture in the posterior free wall had occurred, resulting in a tamponade of $400 \mathrm{ml}$ blood. In addition to the free wall rupture, a partial rupture of the posteromedial papillary muscle group was present (Fig. 4A). The latter could account for the sudden onset of signs of mitral regurgitation. Examination of the coronary arteries showed onevessel disease, with a recent complete occlusion distally in the dominant right coronary artery. There were no signs of previous myocardial infarction. Microscopical studies of the infarct revealed a massive leucocytic infiltration of approximately 3 to 7 days. It was concluded, therefore, that the sudden onset of chest pain 1 day before death probably represented the onset of rupture. It could well be that the period of chest pain experienced 5 days before death and which spontaneously subsided was the moment of onset of infarction.

\section{Discussion}

It has been suggested by Lautsch and Lanks (1967), from a morphological study of heart rupture cases, that features often existed indicating a delayed rupture. These authors concluded, from their correlation between morphological studies and clinical histories, that in some instances the onset of symptoms could have been caused by the onset of rupture. Van Tassel and Edwards (1972), in a recent study of 28 hearts from patients with a free wall rupture, also found evidence to support this concept. Recently the term 'concealed rupture' has been proposed for this phenomenon (Becker and van Mantgem, 1975).

The term is intended for cases where the actual onset of rupture is clinically 'concealed' and interpreted as the onset of infarction. The term is proposed to contrast the common situation in which the dramatic episode of rupture, though unpredicted, is well recognized within the setting of an acute infarction. The observations described here have in common that in each of the 4 instances evidence existed for 'concealed rupture'. This conclusion is based upon a series of considerations.

Firstly, histological examination of the infarcted myocardium shows a pronounced difference in the age of myocardial necrosis. It is, of course, appreciated that an exact time-table of the reparative 


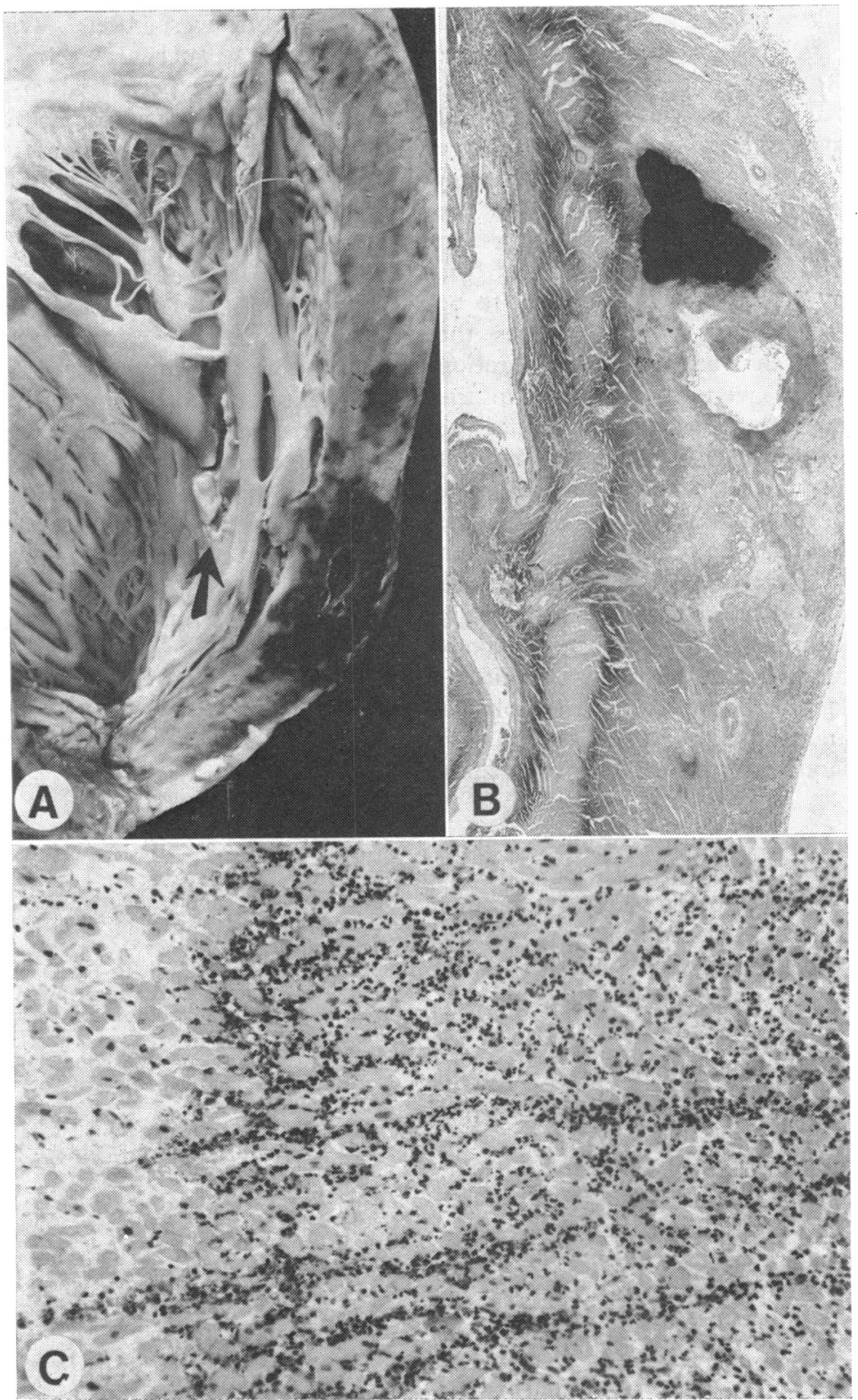

FIG. 4 Case 4. The clinical dating of the infarct was approximately 1 day. (A) Gross aspect of opened left ventricle. The cut surface shows a haemorrhage secondary to the rupture. There was a posteroinferior wall infarct extending into the lateral wall. A partial rupture of the posteromedial papillary muscle group has occurred (arrow). The actual breakthrough is posteriorly and cannot be recognized in this view. (B) Distinct demarcation of dead myocardium by a zone of polymorphonuclear leucocytes. A recent haemorrhage related to the rupture is present in the outer part of the left ventricular wall. (C) A detailed view of a representative microscopical section. The infarct shows massive infiltration with polymorphonuclear leucocytes, indicative of an injury older than 1 day. This lends support to the view that the sudden onset of chest pain one day before death could represent the onset of rupture. (Haematoxylin and eosin. B $\times 4 \cdot 6$, $C \times 95$.) 
responses upon infarction cannot be given. However, it is widely agreed that the invasion of the myocardium by polymorphonuclear leucocytes needs time to evolve. The classical studies of Mallory, White, and Salcedo-Salgar (1939) have shown that the earliest invasion with leucocytes can be expected approximately 24 hours after the onset of infarction. Personal experiences are in agreement with this statement. Therefore, the findings in Cases 1, 3, and 4 are considered to be of significance. In each of these instances the microscopical studies showed massive infiltration with leucocytes, which were also present in the deeper parts of the affected myocardium. Since the peak of leucocytic infiltration is generally thought to occur somewhere between 3 and 7 days after the onset of infarction, the myocardial necrosis in the 3 patients is considered to be of an older 'age' than the clinical history suggests. The same holds true for Case 2 in whom the infarction was clinically considered to be 4 days old, whereas the histology showed early fibroblastic and capillary proliferation. The cellular reaction, i.e. macrophages carrying lipofuchsin and iron pigments, was also in keeping with a reparative response of approximately 1 to 3 weeks.

The second argument is based upon the gross aspect of the actual rupture site. In each of the four instances the site of rupture showed a distinct erosion of the necrotic myocardium. It has been shown that this type of rupture coincides with myocardial infarction of a somewhat longer standing (Becker and van Mantgem, 1975).

Apparently, the 'erosion' needs time, though examples are present where the phenomenon of erosion had occurred in about 24 hours. It is an indication, however, that the tear leading to tamponade is not necessarily a sudden event. Instead, the 'erosion' in our opinion indicates that a considerable time lapse may exist between the onset of the tear and the actual epicardial breakthrough. This endorses the opinion previously expressed by morphologists (Lautsch and Lanks, 1967; Van Tassel and Edwards, 1972; Becker and van Mantgem, 1975) and is in keeping with a number of clinical reports, some of which indicate the possibility of early recognition and surgical intervention (Lillehei et al., 1969; Buckley et al., 1971; Mundth, 1972; Löfström et al., 1972; O'Rourke, 1973; Cobbs, Hatcher, and Robinson, 1973; Calick et al., 1974).

A third argument is based upon the clinical history of the patients. In retrospect, each of the patients experienced symptoms which could well be attributed to the onset of infarction. For various reasons, however, these symptoms were not interpreted as such. It seems likely that each of these patients experienced an infarct, with only minimal or non-specific signs, for some time before admission. Indeed, the high frequency of undiagnosed or clinically 'silent' infarction among a large necropsy series has previously been emphasized (Melichar, Jedlicka, and Havlik, 1963).

What then caused the sudden onset of symptoms? The possibility of recurrent infarction should be considered. However, the postmortem studies did not support this supposition. In each instance the tear went through the infarct, which itself showed the lack of correlation with the clinical history as discussed previously. The aspect of the tear, moreover, endorses the view that the process of rupturing took some considerable time. This, then, leads to the concept that the moment of sudden pain, interpreted as the onset of an infarction, represented the onset of rupture.

We are grateful to our clinical colleagues and in particular to Dr. K. I. Lie, Department of Cardiology, Wilhelmina Gasthuis, Amsterdam, for his help in evaluating the clinical records.

\section{References}

Becker, A. E., and van Mantgem, J-P. (1975). Cardiac tamponade. A study of 50 hearts. European fournal of Cardiology, 3, 349.

Buckley, M. J., Mundth, E. D., Daggett, W. M., DeSanctis, R. W., Sanders, C. A., and Austen, W. G. (1971). Surgical therapy for early complications of myocardial infarction. Surgery, 70, 814 .

Calick, A., Kerth, W., Barbour, D., and Cohn, K. (1974). Successful surgical therapy of ruptured myocardium. Chest, 66, 188.

Cobbs, B. W., Jr., Hatcher, C. R., Jr., and Robinson, P. H. (1973). Cardiac rupture. Three operations with two longterm survivals. Fournal of the American Medical Association, 223, 532.

Lautsch, E. V., and Lanks, K. W. (1967). Pathogenesis of cardiac rupture. Archives of Pathology, 84, 264.

Lie, K. I., Roels-van Ijsseldijk, Y. C., and Van Capelle, F. J. L. (1975). Factoren die de prognose van het acute hartinfarct beinvloeden. Nederlandsch Tijdschrift voor Geneeskunde, 119, 466.

Lillehei, C. W., Lande, A. J., Rassman, W. R., Tanaka, S., and Block, J. H. (1969). Surgical management of myocardial infarction. Some promising concepts utilizing revascularization, mechanical circulatory assistance, operative treatment of severe complications, and cardiac replacement. Circulation, 39-40 (Suppl. IV), 315.

Löfström, B., Mogensen, L., Nyquist, O., Orinius, E., Sjögren, A., and Werner, B. (1972). Studies of myocardial rupture with cardiac tamponade in acute myocardial infarction. III. Attempts at emergency surgical treatment. Chest, 61, 10.

Mallory, G. K., White, P. D., and Salcedo-Salgar, J. (1939). The speed of healing of myocardial infarction. A study of the pathologic anatomy in seventy-two cases. American Heart fournal, 18, 647 .

Melichar, F., Jedlicka, V., and Havlik, L. (1963). A study of undiagnosed myocardial infarctions. Acta Medica Scandinavica, 174, 761. 
Mundth, E. D. (1972). Rupture of the heart complicating myocardial infarction. Circulation, 46, 427.

O'Rourke, M. F. (1973). Subacute heart rupture following myocardial infarction. Clinical features of a correctable condition. Lancet, $2,124$.

Van Tassel, R. A., and Edwards, J. E. (1972). Rupture of heart complicating myocardial infarction. Analysis of $\mathbf{4 0}$ cases including nine examples of left ventricular false aneurysm. Chest, 61, 104.

Requests for reprints to Dr. A. E. Becker, Department of Pathology, Wilhelmina Gasthuis, Eerste Helmersstraat 104, Amsterdam, The Netherlands. 